\title{
Imaging characteristics of esophageal cancer in multi-slice spiral CT and barium meal radiography and their early diagnostic value
}

\author{
Jiafu Wang ${ }^{1 \#}$, Liang Tang ${ }^{2 \#}$, Lin $\operatorname{Lin}^{1}$, Yanyan $\mathrm{Li}^{3}, \mathrm{Jin}^{{ }^{4}}{ }^{4}$, Wenbo $\mathrm{Ma}^{3}$ \\ ${ }^{1}$ Department of Nuclear Medicine, The First Affiliated Hospital of Harbin Medical University, Harbin, China; ${ }^{2}$ Department of CT, The First \\ Affiliated Hospital of Harbin Medical University, Harbin, China; ${ }^{3}$ Department of Radiology, The First Affiliated Hospital of Harbin Medical \\ University, Harbin, China; ${ }^{4}$ Department of Integrative Medicine Oncology, The First Affiliated Hospital of Harbin Medical University, Harbin, \\ China \\ Contributions: (I) Conception and design: J Wang, L Tang; (II) Administrative support: Y Li, W Ma; (III) Provision of study materials or patients: L \\ Tang, L Lin; (IV) Collection and assembly of data: J Wang, Y Li, J Li; (V) Data analysis and interpretation: J Wang, L Tang, L Lin; (VI) Manuscript \\ writing: All authors; (VII) Final approval of manuscript: All authors. \\ "These authors contributed equally to this work. \\ Correspondence to: Jiafu Wang. Department of Nuclear Medicine, The First Affiliated Hospital of Harbin Medical University, 23 Youzheng Street, \\ Nangang District, Harbin 150001, China. Email: wangjiafu20212021@163.com.
}

Background: To explore the imaging characteristics of esophageal cancer in multi-slice spiral CT (MSCT) and barium meal radiography and to analyze the value of the two examinations alone or in combination for cancer staging diagnosis.

Methods: The clinical data of 87 patients with esophageal cancer admitted to our hospital from June 2018 to June 2020 were retrospectively analyzed. According to the different examination methods, they were divided into a barium meal group ( $\mathrm{n}=28$ cases, X-ray barium meal radiography), an MSCT group ( $n=29$ cases, MSCT examination), and a combined group ( $\mathrm{n}=30$ cases, barium meal + MSCT). The imaging characteristics from the barium meal radiography and MSCT alone versus their combined results were compared with the pathological examination results to analyze their accuracy in diagnosing esophageal cancer staging.

Results: Of the 87 cases, the esophageal cancer lesion sites were as follows: 23 cervical cases, 20 upper thoracic cases, 21 middle thoracic cases, and 23 lower thoracic cases. The X-ray barium meal examination of esophageal mucosa showed irregular filling of the esophagus. The esophageal wall was stiff or jagged, the lumen was narrow, and it was difficult for the barium to pass, the mucosa was discontinuous. The CT scan showed irregular thickening of the esophageal wall, eccentric and concentric stenosis of the esophageal cavity, and the upper part of the esophagus showed different degrees of expansion. The trachea and bronchi were invaded, deformed, and displaced under compression. The diagnostic staging results of the barium meal group and MSCT group were inconsistent with the pathological results (Kappa =0.105, 0.112; P>0.05). The diagnostic staging results of the combined group were in good agreement with the pathological results (Kappa $=0.769$ ). In addition, the accuracy of the combined group in the diagnosis of stage III-IV esophageal cancer among the three groups was significantly higher than that of the barium meal group and MSCT group alone $(\mathrm{P}<0.05)$.

Conclusions: MSCT and barium meal radiography clearly display the imaging features of esophageal cancer and can provide reliable imaging evidence for preoperative diagnosis; the combination of both measures can effectively improve the accuracy of early diagnosis for esophageal cancer.

Keywords: Multi-slice spiral CT (MSCT); X-ray barium meal radiography; esophageal cancer; clinical staging

Submitted Dec 02, 2021. Accepted for publication Feb 11, 2022.

doi: 10.21037/jgo-22-36

View this article at: https://dx.doi.org/10.21037/jgo-22-36 


\section{Introduction}

Esophageal cancer is a malignant tumor occurring in esophageal epithelial tissue, accounting for $2 \%$ of all malignant tumors, with high morbidity and mortality (1). The incidence of male is higher than female, and the age of onset is more than 40 years old Most patients have no specific clinical symptoms in the early stage of the disease, which is characterized by a high risk of lymph node metastasis. Therefore, the optimal treatment time has often been missed by the time of initial clinical diagnosis, which adversely affects patients' long-term survival. Moreover, patients with esophageal cancer often have symptoms such as pharyngeal choking, progressive pharyngeal difficulty, and persistent chest or back pain, which can severely impact their physical and mental health $(2,3)$. Surgical resection is the primary clinical treatment for esophageal cancer. The resection rate is closely related to the accuracy of the preoperative diagnosis. A clear understanding of the lesion's size, location, scope, and metastasis is the key to successful surgical treatment $(4,5)$. Therefore, it is crucial to have effective clinical examination methods to diagnose esophageal cancer accurately. MRI, barium meal and multi-slice spiral CT (MSCT) are commonly used in clinical diagnosis of esophageal cancer. MRI has some limitations due to its long examination time and high cost. At present, barium meal or MSCT is often used in early clinical diagnosis, but the diagnostic value of the two is still controversial. For this reason, we retrospectively analyzed the clinical data of 87 patients with esophageal cancer. Barium meal radiography and MSCT examinations were evaluated alone and in combination to determine their clinical value in providing reliable guidance for clinical diagnosis and treatment. We present the following article in accordance with the STROBE reporting checklist (available at https://jgo.amegroups.com/article/ view/10.21037/jgo-22-36/rc).

\section{Methods}

\section{General information}

The clinical data of patients with esophageal cancer admitted to our hospital from June 2018 to June 2020 were retrospectively analyzed. The inclusion criteria were as follows: (I) all patients were required to meet the diagnostic criteria for esophageal cancer as specified in the "Guidelines for standardized diagnosis and treatment of esophageal cancer" (6); (II) patients were over 18 years old with complete clinical data; (III) no other malignant tumors were present; (IV) patients were not receiving radiotherapy or chemotherapy. Patients were excluded for the following reasons: (I) any previous barium meal, CT examination, or preoperative radiotherapy and chemotherapy-related treatment; (II) patients with incomplete data; (III) patients with coagulation disorders; (IV) patients with esophageal cancer recurrence. All procedures performed in this study involving human participants were in accordance with the Declaration of Helsinki (as revised in 2013). The study was approved by ethics committee of The First Affiliated Hospital of Harbin Medical University [2020GS42 (Research 2019132)]. Individual consent for this retrospective analysis was waived.

A total of 87 patients with esophageal cancer were included and divided into three groups according to the different examination methods. There were 28 cases in the barium meal group, 29 cases in the MSCT group, and 30 cases in the combined group. There was no difference in the general demographic data of the three groups, which were comparable $(\mathrm{P}>0.05)$. See Table 1.

\section{Study methods}

\section{Barium meal group}

This group of patients was examined with a barium meal $\mathrm{X}$-ray. The inspection instrument was an Sonialvision Versa $80 \mathrm{X}$-ray machine (Shimadzu, Japan). Patients were placed in the oblique and vertical position and instructed to swallow water mixed with type II barium sulfate with a 1:3 ratio of water to barium. Simultaneous dual radiography was performed with air and barium.

\section{MSCT group}

The patients in this group were examined by MSCT using a 256-slice spiral Brilliance iCT machine (Philips, Netherlands). The patient was placed in the supine position. The scanning range was from the thoracic entrance of the lower neck to the fundus of the stomach. The scanning parameters were as follows: tube voltage $120 \mathrm{kV}$, tube current $210 \mathrm{~mA}$, scanning layer thickness $5 \mathrm{~mm}$, pitch 1.0, and reconstruction layer thickness $2 \mathrm{~mm}$. Both conventional and enhanced scans were conducted. During the enhanced scan, $80 \mathrm{~mL}$ of iohexol was injected through the patient's elbow at a rate of $3.0 \mathrm{~mL} / \mathrm{s}$.

\section{Combined group}

The patients in this group were examined by barium meal radiography combined with MSCT scanning. The specific conditions and related procedures are outlined above. 
Table 1 Comparison of general between-group data

\begin{tabular}{|c|c|c|c|c|c|c|}
\hline Group & $\begin{array}{c}\text { Gender } \\
\text { (male/female) }\end{array}$ & $\begin{array}{c}\text { Average age } \\
\text { (years), } \bar{x} \pm s\end{array}$ & $\begin{array}{l}\text { Tumor diameter } \\
\text { (cm), } \bar{x} \pm \mathrm{S}\end{array}$ & \multicolumn{3}{|c|}{ Tumor type, n (\%) } \\
\hline MSCT group $(n=29)$ & $14 / 15$ & $64.96 \pm 6.71$ & $4.11 \pm 1.13$ & $16(55.17)$ & $7(24.14)$ & $6(20.69)$ \\
\hline$P$ & 0.778 & 0.848 & 0.898 & & 0.973 & \\
\hline
\end{tabular}

MSCT, multi-slice spiral CT.

\section{Observation indicators}

(I) The imaging examination results of 87 patients were recorded. Two or more senior radiologists in our department read the results using the double-blind method. When disagreements occurred, the final results were determined through consultation. The size, shape, and lymph node metastasis of malignant lesions were observed. (II) The results of the barium meal radiography and MSCT examinations alone and in combination were compared with the pathological staging results, and the accuracy of the esophageal cancer staging was calculated. Esophageal cancer staging was established according to the "Interpretation and evaluation of the 7th edition of international TNM staging of esophageal cancer" (7) as follows: stage I, localized esophageal wall or luminal mass thickening $\leq 5 \mathrm{~mm}$; stage II, esophageal wall thickening $>5 \mathrm{~mm}$ but no invasion or distant metastasis; stage III, esophageal wall thickening $>5 \mathrm{~mm}$, invasion of the surrounding esophagus, enlarged mediastinal lymph nodes but no distant metastasis; stage $\mathrm{IV}$, esophageal wall thickening $>5 \mathrm{~mm}$, invasion of the surrounding esophagus, and distant metastasis.

\section{Statistical method}

The data in this study were statistically analyzed by SPSS 18.0 software (IBM Corp., Chicago, IL, USA). Continuous variables were described by the mean \pm standard deviation $\left(\bar{x}_{ \pm \mathrm{s}}\right)$. The $t$-test was used to compare two groups, and the variance test was used to compare multiple groups. Categorical variables were expressed by rate or composition ratio and analyzed with the $\chi^{2}$ test. The Kappa test was used for the consistency analysis. A $\mathrm{P}$ value $<0.05$ indicated that the difference was statistically significant.

\section{Results}

\section{Pathological diagnosis results}

The 87 patients were staged as follows: 54 cases in stage I-II and 33 cases in stage III-IV. In the barium meal group, there were 17 cases in stage I-II and 11 cases in stage III-IV. In the MSCT group, there were 16 cases in stage I-II and 13 cases in stage III-IV. The combined group consisted of 21 cases in stage I-II and 9 cases in stage III-IV.

\section{Comparison of staging results and pathological examination results in the different groups}

As shown in Table 2, there was poor consistency between the staging diagnosis results and the pathological examination results in the barium meal and MSCT groups (Kappa $=0.105,0.112 ; \mathrm{P}>0.05)$. The staging diagnosis of the combined group was consistent with the pathological results $($ Kappa $=0.769)$.

\section{Comparison of diagnostic staging accuracy between the different groups}

The accuracy rates of the barium meal group in the diagnosis of stage I-II and stage III-IV esophageal cancer were $82.35 \%$ and $27.27 \%$, respectively. The MSCT group was $87.50 \%$ and $23.08 \%$, respectively. The combined group was $90.48 \%$ and $88.89 \%$, respectively. There was no significant difference in the accuracy of the three groups in the diagnosis of stage I-II esophageal cancer $(\mathrm{P}>0.05)$. However, the accuracy of the combined group in the diagnosis of stage III-IV esophageal cancer was significantly higher than that of the barium meal and MSCT groups, and the difference was statistically significant $(\mathrm{P}<0.05)$. See Table 3. 
Table 2 Comparison of staging and pathological examination results in different groups

\begin{tabular}{|c|c|c|c|c|c|c|}
\hline Group & Staging & \multicolumn{2}{|c|}{ Pathological results, n (\%) } & Total & Youden index & Kappa \\
\hline \multirow[t]{2}{*}{ Barium meal group } & Stage I-II & $14(82.35)$ & $8(72.73)$ & 22 & 0.097 & 0.105 \\
\hline & Stage III-IV & $3(17.65)$ & $3(27.27)$ & 6 & & \\
\hline MSCT group & Stage I-II & $14(87.50)$ & $10(76.92)$ & 24 & 0.106 & 0.112 \\
\hline \multirow[t]{2}{*}{ Combined group } & Stage I-II & $19(90.48)$ & $1(11.11)$ & 20 & 0.794 & 0.769 \\
\hline & Stage III-IV & $2(9.52)$ & $8(88.89)$ & 10 & & \\
\hline
\end{tabular}

MSCT, multi-slice spiral CT.

Table 3 Comparison of diagnostic staging accuracy among different groups

\begin{tabular}{lcc}
\hline Group & Stage I-II $(\%)$ & Stage III-IV (\%) \\
\hline Barium meal group $(\mathrm{n}=28)$ & 82.35 & 27.27 \\
MSCT group $(\mathrm{n}=29)$ & 87.50 & 23.08 \\
Combined group $(\mathrm{n}=30)$ & 90.48 & 88.89 \\
$\chi^{2}$ & 0.554 & 10.981 \\
P & 0.758 & 0.004 \\
\hline
\end{tabular}

MSCT, multi-slice spiral CT.

\section{Imaging signs}

\section{Lesions}

There were 87 cases of esophageal carcinoma lesions: 23 cervical cases, 20 upper thoracic cases, 21 middle thoracic cases, and 23 lower thoracic cases. The X-ray barium meal signs, MSCT and pathological examination results of a 66-year-old male esophageal squamous cell carcinoma, as shown in Figure 1.

\section{$\mathrm{X}$-ray barium meal signs}

(I) The esophageal mucosa demonstrated visible irregular thickening and disorder; (II) the esophageal filling was irregular; (III) the esophageal wall was rigid or serrated; (IV) the lumen was narrow, making it difficult for the barium to pass; $(\mathrm{V})$ other signs included tracheal compression, tracheoesophageal fistula, and mediastinal widening.

\section{MSCT signs}

(I) Direct signs included visible local irregular thickening of the esophageal wall, esophageal eccentric and concentric stenosis, varying degrees of expansion visible in the upper esophageal tract; (II) indirect signs included the invasion of fat space around the lesion, surrounded by only a fat layer without serosa; tracheal and bronchial invasion, compression, deformation, and displacement. Soft tissue masses were seen in some lumens; the aortic arch, thoracic aorta, pleura, and pericardium were invaded. The enhanced scan showed mild to moderate heterogeneous enhancement.

\section{Discussion}

Esophageal cancer is a common malignant tumor of the digestive tract with high mortality. The occurrence of esophageal cancer has a noticeable regional distribution. There are approximately 314,000 new cases in the world every year, of which about 167,000 are found in China, where esophageal cancer is ranked fourth in malignant tumor incidence. And esophageal cancer is ranked second in malignant tumors of the digestive tract, only slightly lower than lung cancer $(8,9)$. Early onset of esophageal cancer is relatively hidden, with mild and non-specific symptoms. Because the symptoms are intermittent and are vulnerable to dietary and emotional factors, they are often ignored (10). Studies have shown that advanced esophageal cancer accounts for more than $90 \%$ of cases. The survival rate of advanced esophageal cancer is low, at less than 3\% (11). Therefore, early detection, early diagnosis, and early treatment are critical for esophageal cancer patients and can effectively improve their prognosis and survival rate.

There are many traditional examination methods for diagnosing esophageal cancer, including esophageal mucosal cytology and fibro-esophageal endoscopy. However, long-term studies have confirmed that such examinations can cause varying degrees of trauma, making them less acceptable to patients $(12,13)$. At present, 

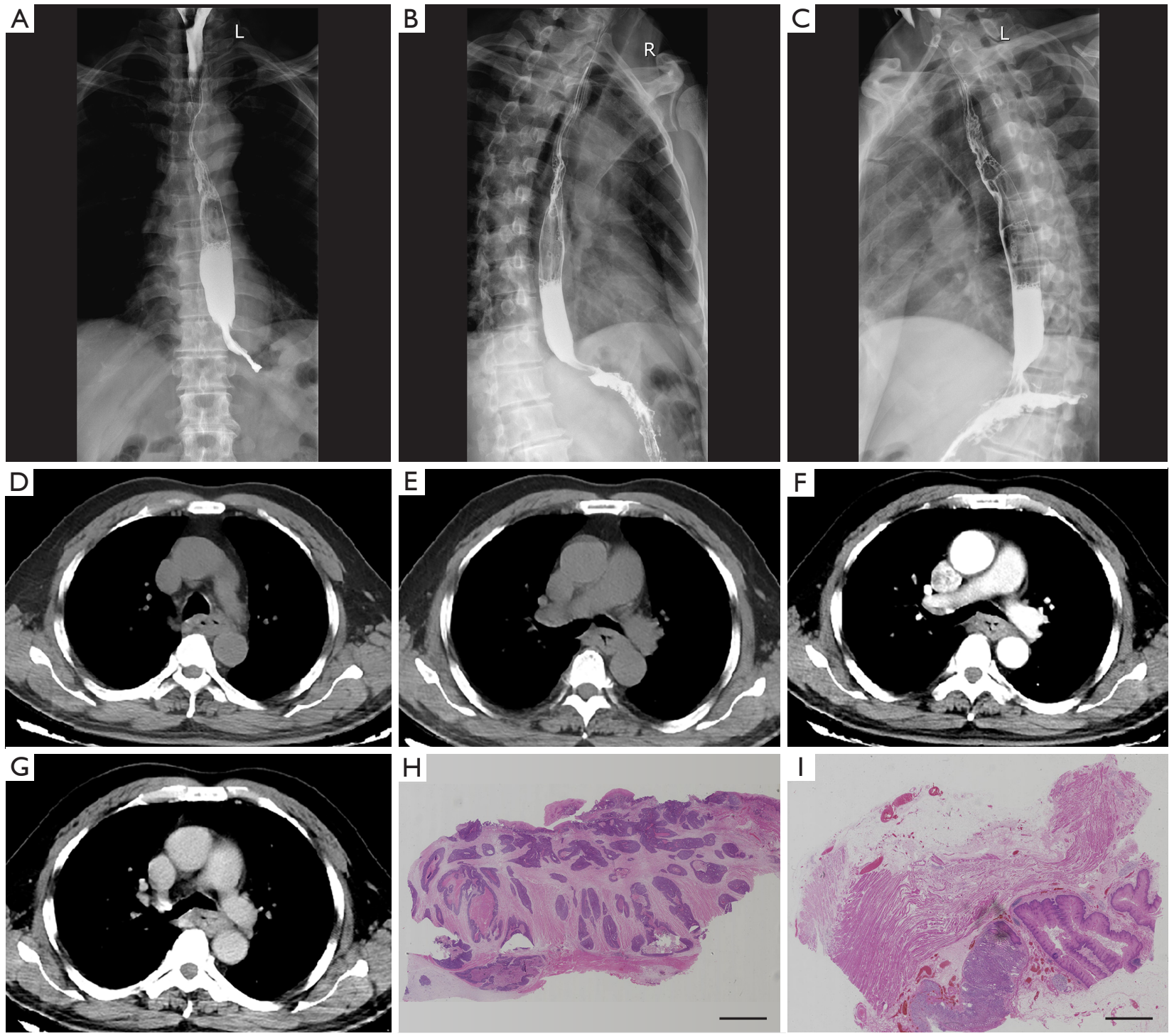

Figure 1 A 66-year-old male presented with progressive dysphagia for 3 months. X-ray barium meal angiography of the upper gastrointestinal shows that the middle thoracic esophageal lumen is narrow, stiff, and has mucosal disorder, and the upper esophagus is slightly dilated (A-C). The CT plain scan of the middle part of the thoracic esophageal wall shows thickening and lumen stenosis (D,E). The enhanced scan shows noticeable continuous enhancement (F,G). HE staining pathology: poorly differentiated squamous cell carcinoma (H) and ulcerated-type moderately differentiated squamous cell carcinoma (I). Scale bar, $5 \mathrm{~mm}$. HE, hematoxylin-eosin.

the primary clinical diagnostic methods for esophageal cancer are MSCT and X-ray barium meal. The above two methods are noninvasiveness and simple procedures. Barium meal radiography can dynamically observe the location, morphology, esophageal mucosa, and cavity wall of esophageal tumors in real-time and is particularly accurate for the early diagnosis of small lesions. MSCT scanning can clearly show the location, size, and scope of esophageal tumors and the surrounding tissues and organs. MSCT can accurately measure the thickness of the esophageal wall and determine the scope of the tumor invasion and lymph node metastasis, which is of great help in the clinical staging and prognosis of esophageal cancer $(14,15)$. In this study, it was observed that CT diagnosis of esophageal cancer was not difficult, and most scans showed a soft tissue mass or concentric circular thickening of the lumen 
with lumen stenosis and local wall thickening with lumen stenosis. In addition, tumors are prone to directly invade adjacent structures due to the absence of serous membrane outside the esophagus. In our patients, the most frequently invaded organs were the aorta, trachea, bronchus, lung, and pericardium. The mucosal and gastrointestinal motility and morphological changes in the lesion area could be observed by barium meal examination. The results showed that barium meal and MSCT can provide a clear imaging basis for the clinical diagnosis of esophageal cancer.

However, long-term studies have confirmed that barium meal radiography and MSCT have shortcomings. Barium meal radiography cannot effectively display the surrounding structure and invasion of the esophagus and has other disadvantages, such as inaccurate observation of tumor morphology, insufficient range, and inaccurate staging results. And MSCT scans cannot display superficial mucosal lesions, so they also suffer from inaccurate staging results (16). However, the combination of barium meal and MSCT can significantly improve the value of early diagnosis in esophageal cancer. For example, barium meal angiography as the first choice for observation and screening can effectively show the esophageal tumor, mucosa, and cavity wall. MSCT can clearly show the maximum width of the lesion, the distance between the midline of the spinal cord and the related parts, the degree of invasion and mediastinal lymph node metastasis. The application of MSCT scanning in the diagnosis of esophageal cancer can well supplement the insufficient barium meal angiography. The combination of the two can provide more abundant imaging information for the diagnosis of esophageal cancer and improve the diagnostic value (17). This study showed that the consistency of the combined group diagnostic staging results with the pathological results was significantly better than that of the barium meal or MSCT groups alone. The Youden index for the combined group was also higher than that of the barium meal or MSCT groups. We observed no significant difference in the accuracy of the three groups when diagnosing stage I-II esophageal cancer $(\mathrm{P}>0.05)$. However, the accuracy of the combined group in the diagnosis of stage III-IV esophageal cancer was significantly higher than that of the barium meal group or MSCT group alone. It indicates that a combination of the two examinations has a higher value in clinical use, consistent with the research reports of Pietrzak et al. (18).

In summary, MSCT and barium meal clearly show the imaging features of esophageal cancer and can provide a reliable imaging basis for preoperative diagnosis.
Combining the two can effectively improve the accuracy of the early diagnosis of esophageal cancer.

\section{Acknowledgments}

Funding: None.

\section{Footnote}

Reporting Checklist: The authors have completed the STROBE reporting checklist Available at https://jgo. amegroups.com/article/view/10.21037/jgo-22-36/rc

Data Sharing Statement: Available at https://jgo.amegroups. com/article/view/10.21037/jgo-22-36/dss

Conflicts of Interest: All authors have completed the ICMJE uniform disclosure form (available at https://jgo.amegroups. com/article/view/10.21037/jgo-22-36/coif). The authors have no conflicts of interest to declare.

Ethical Statement: The authors are accountable for all aspects of the work in ensuring that questions related to the accuracy or integrity of any part of the work are appropriately investigated and resolved. All procedures performed in this study involving human participants were in accordance with the Declaration of Helsinki (as revised in 2013). The study was approved by ethics committee of The First Affiliated Hospital of Harbin Medical University [2020GS42 (Research 2019132)]. Individual consent for this retrospective analysis was waived.

Open Access Statement: This is an Open Access article distributed in accordance with the Creative Commons Attribution-NonCommercial-NoDerivs 4.0 International License (CC BY-NC-ND 4.0), which permits the noncommercial replication and distribution of the article with the strict proviso that no changes or edits are made and the original work is properly cited (including links to both the formal publication through the relevant DOI and the license). See: https://creativecommons.org/licenses/by-nc-nd/4.0/.

\section{References}

1. Zhang QY, Dong XY, Zang NL, et al. Advances in research and application of CRISPR/Cas9 system in human diseases. Journal of Molecular Diagnosis and Therapy 2018;10:206-11. 
2. Odawara S, Kitajima K, Katsuura T, et al. Tumor response to neoadjuvant chemotherapy in patients with esophageal cancer assessed with CT and FDG-PET/CT - RECIST 1.1 vs. PERCIST 1.0. Eur J Radiol 2018;101:65-71.

3. Fu YK, Chen ZX. Expressions and Clinical Significances of T-lymphocyte Subsets and Bcl-2 Protein in Esophageal Carcinoma Tissues. Medical \& Pharmaceutical Journal of Chinese People's Liberation Army 2018;30:28-31, 43.

4. Sturm EC, Zahnd W, Mellinger JD, et al. National trends in local excision and esophagectomy for cT1N0 esophageal cancer. J Clin Oncol 2017;35:abstr 62.

5. Dang J, Zhang YG. Prognostic Value of EZH2 Protein Expression in Post-operative Pa-tients with Esophageal Carcinoma. Journal of Medical Molecular Biology 2018;15:151-2.

6. Hao YY, Cheng LL, Yu XF. Efficacy and safety of concurrent chemoradiotherapy combined with Taixinsheng in the treatment of advanced esophageal cancer. Health Medicine Research and Practice 2019;16:58-62, 69.

7. Voncken FEM, Vegt E, Sandick JW, et al. MotionCompensated FDG PET/CT for Esophageal Cancer. Int J Radiat Oncol Biol Phys 2019;105:E200.

8. Thuss-Patience P, Vecchione L, Keilholz U. Should cT2 esophageal cancer get neoadjuvant treatment before surgery? J Thorac Dis 2017;9:2819-23.

9. Rosenwald KD, Hayes K, Menard-Katcher C, et al. Implementation of a Timed Barium Esophagram Protocol for Assessment of Esophageal Function in Children. J Pediatr Gastroenterol Nutr 2020;71:470-5.

10. Posner S, Mehta K, Parish A, et al. Esophageal Function Tests are Not Associated with Barium Swallow Findings in Advanced Lung Disease. Dysphagia 2020;35:864-70.

11. Posner S, Mehta K, Parish A, et al. Correction to:

Cite this article as: Wang J, Tang L, Lin L, Li Y, Li J, Ma W. Imaging characteristics of esophageal cancer in multi-slice spiral CT and barium meal radiography and their early diagnostic value. J Gastrointest Oncol 2022;13(1):49-55. doi: 10.21037/jgo22-36
Esophageal Function Tests are Not Associated with Barium Swallow Findings in Advanced Lung Disease. Dysphagia 2020;35:871.

12. Stavropoulos SN, Gurram KC, Bhumi S, et al. Tu1259 ESD for Early Esophageal Cancer at a Western Center: Practice Makes Perfect. Gastrointestinal Endoscopy 2017;85:AB605-6.

13. Tassi V, Lugaresi M, Mattioli B, et al. Incidence and risk factors for the development of epidermoid carcinoma in oesophageal achalasia†. Eur J Cardiothorac Surg 2019;55:956-63.

14. Smit JK, Muijs CT, Burgerhof JG, et al. Survival after definitive (chemo)radiotherapy in esophageal cancer patients: a population-based study in the north-East Netherlands. Ann Surg Oncol 2013;20:1985-92.

15. Goense L, Heethuis SE, van Rossum PSN, et al. Correlation between functional imaging markers derived from diffusion-weighted MRI and 18F-FDG PET/CT in esophageal cancer. Nucl Med Commun 2018;39:60-7.

16. Larue RTHM, Klaassen R, Jochems A, et al. Pretreatment CT radiomics to predict 3-year overall survival following chemoradiotherapy of esophageal cancer. Acta Oncol 2018;57:1475-81.

17. Melsens E, De Vlieghere E, Descamps B, et al. Hypoxia imaging with $18 \mathrm{~F}-\mathrm{FAZA}$ PET/CT predicts radiotherapy response in esophageal adenocarcinoma xenografts. Radiat Oncol 2018; 13:39.

18. Pietrzak AK, Martenka P, Strzesak E, et al. Will Rogers phenomenon in the oesophageal cancer patients staging CT versus 18F-FDG PET/CT: retrospective study. Hell J Nucl Med 2019;22 Suppl 2:174-80.

(English Language Editor: D. Fitzgerald) 